See discussions, stats, and author profiles for this publication at: https://www.researchgate.net/publication/319999378

\title{
Assessing the clinical outcome of Vim radiosurgery with voxel-based morphometry: visual areas are linked with tremor arrest!
}

Article in Acta Neurochirurgica · September 2017

DOI: 10.1007/s00701-017-3317-7

CITATIONS

23

12 authors, including:

Constantin Tuleasca

Centre Hospitalier Régional Universitaire de Lille

365 PUBLICATIONS 565 CITATIONS

SEE PROFILE

Nadine Girard

Assistance Publique Hôpitaux de Marseille

421 PUBLICATIONS 4,773 CITATIONS

SEE PROFILE

Some of the authors of this publication are also working on these related projects:

Project Book about Gamma Knife Radiosurgery (Russian). Edited by prof. Andrey V. Golanov and Valery V. Kostjuchenko View project

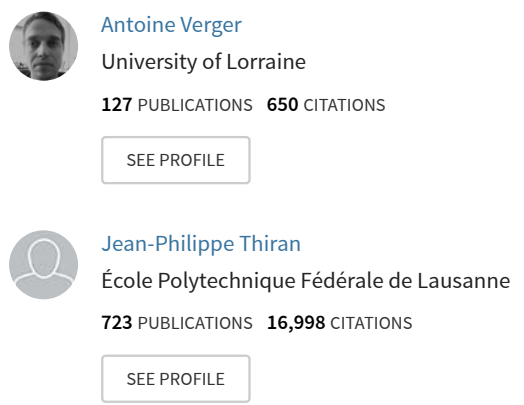

\footnotetext{
Project $\quad$ OCD and Gamma Knife View project
} 


\section{Assessing the clinical outcome of Vim radiosurgery with voxel-based morphometry: visual areas are linked with tremor arrest!}

Constantin Tuleasca ${ }^{1,3,9}$, MD, Tatiana Witjas ${ }^{4,5}$, MD, Elena Najdenovska ${ }^{2}$, MSc, Antoine Verger $^{6}$, MD, Nadine Girard ${ }^{7}$, MD-PHD, Jerome Champoudry ${ }^{8}$, MSc, Jean-Philippe Thiran ${ }^{3,9}$, PHD, Dimitri van de Ville ${ }^{11}$, PHD, Meritxell Bach Cuadra ${ }^{2}$, PHD, Marc Levivier ${ }^{1,9}$, MD, PHD, Eric Guedj ${ }^{6}$, MD, AND Jean Régis ${ }^{8}$, MD

${ }^{1}$ Centre Hospitalier Universitaire Vaudois (CHUV), Neurosurgery Service and Gamma Knife Center, Lausanne, Switzerland; ${ }^{2}$ Radiology Department, Center for Biomedical Imaging (CIBM), Lausanne University Hospital (CHUV) and University of Lausanne (UNIL), Centre Hospitalier Universitaire Vaudois, Lausanne, Switzerland;

${ }^{3}$ Signal Processing Laboratory (LTS 5), Ecole Polytechnique Fédérale de Lausanne (EPFL), Switzerland;

${ }^{4}$ Department of Neurology and Movement Disorders, Assistance Publique-Hôpitaux de Marseille, Aix-Marseille Université, Timone University Hospital, France; ${ }^{5}$ Institut de Neurosciences de la Timone, UMR 7289, CNRS Aix-Marseille Université, Marseille, France; ${ }^{6}$ Department of Nuclear Medicine, Assistance Publique-Hôpitaux de Marseille, Aix-Marseille Université, Timone University Hospital, France;Institut de Neurosciences de la

Timone, UMR 7289, CNRS Aix-Marseille Université, Marseille, France;CERIMED, Aix-Marseille Université, Marseille, France; ${ }^{7}$ AMU, CRMBM UMR CNRS 7339, Faculté de Médecine et APHM, Hopital Timone,

Department of Diagnostic and Interventionnal Neuroradiology, Marseille; ${ }^{8}$ Stereotactic and Functional

Neurosurgery Service and Gamma Knife Unit, CHU Timone, Marseille, France; ${ }^{9}$ University of Lausanne,

Faculty of Biology and Medicine; ${ }^{10}$ Medical Image Processing Laboratory, Ecole Polytechnique Fédérale de Lausanne (EPFL), Switzerland ; ${ }^{11}$ University of Geneva, Faculty of Medicine

* the authors equally contributed to the present study

\section{Corresponding author:}

Constantin Tuleasca, MD, MD-PHD candidate, Centre Hospitalier Universitaire Vaudois, Neurosurgery Service and Gamma Knife Center, Rue du Bugnon 44-46, BH-08, CH-1011, Lausanne, Switzerland; Tel: +41-21-314-26-02; Fax: +41-21-314-11-99; e-mail: constantin.tuleasca@gmail.com 


\begin{abstract}
Introduction: Radiosurgery (RS) is an alternative to open standard stereotactic procedures (deep-brain stimulation or radiofrequency thalamotomy) for drug-resistant essential tremor (ET), aiming at same target (ventro-intermediate nucleus, Vim). We investigated Vim RS outcome using voxel-based morphometry, by evaluating interaction between clinical response and time.
\end{abstract}

Methods: Thirty-eight patients with right-sided ET benefited from left unilateral Vim RS. Targeting was performed using $130 \mathrm{~Gy}$ and single 4-mm collimator. Neurological and neuroimaging assessment was completed at baseline and 1 year. Clinical responders were considered those with at least 50\% improvement in tremor score on treated hand (TSTH).

Results: Interaction between clinical response and time showed left temporal pole and occipital cortex (Brodmann area 19, including V4, V5 and parahippocampal place area) as statistically significant. A decrease in grey matter density (GMD) 1 year after Vim RS correlated with higher TSTH improvement (Spearmann= 0.01) for both anatomical areas. Higher baseline GMD within left temporal pole correlated with better TSTH improvement $($ Spearmann $=0.004)$.

Conclusions: Statistically significant structural changes in relationship to clinical response after Vim RS are present in remote areas, advocating a distant neurobiological effect. The former regions are mainly involved in locomotor monitoring towards local and distant environment, suggesting requirement to recruit in targeting specific visuomotor networks.

Key words: tremor, ventro-intermediate nucleus, voxel-based morphometry, Gamma Knife surgery, radiosurgery 


\section{INTRODUCTION}

Essential tremor (ET) is gradually progressive movement disorder, characterized mainly by kinetic and postural tremor, affecting head, forearms and hands[17]. Recent clinical and neuroimaging studies have suggested cerebellar involvement and mediation of tremor by cerebello-thalamo-cortical (CTC) loop, but including also multiple other anatomical regions within the basal ganglia (basal ganglia loop) and cortex[5, 6].

Pharmacological treatment is in a vast majority of cases unsatisfactory. Drug-resistant ET can benefit from standard deep brain stimulation (DBS) or radiofrequeny thermocoagulation or alternatively from minimally invasive radiosurgery (RS) and highfocused ultrasound (HIFU), aiming at the same target (ventro-intermediate nucleus, Vim)[3, 12, 14, 30]. Radiosurgical targeting is indirect and clinical effect installs progressively, over mean period of months, up to one year[30], unlike open surgery, with immediate tremor stop. It has been previously hypothesized that, beside a lesionning effect, Vim RS might have a neuromodulatory one[24].

Neuroimaging procedures have given new insight onto the pathophysiology of tremor [27]. Computational approaches for analyzing high-resolution structural MRI provide a powerful and non-invasive tool for characterizing individual and/or group differences in brain anatomy[1]. With regard to structural changes, voxel-based morphometry (VBM) has been used both at 1.5 and 3 Tesla (T), including in ET, with large spectrum of controversial findings[2, 4, 7-10, 16, 21, 23]. In fact, VBM assesses subtle regional differences, between subjects, in white or gray matter (GM), by a well-established and robust methodology[1].

In the present study, we used VBM to investigate whether Vim RS induces structural gray matter density (GMD) transformations one year after the intervention, in relationship with clinical effect. We hypothesize that changes in remote areas relate to clinical response after Vim RS, beside the targeted site (e.g. left thalamus).

\section{METHODS}

\section{Subjects}

We analyze 38 patients (mean age 71.8 years, range 52-80) with severe refractory right-dominant in right-handed ET, treated with unilateral left Vim RS, between April 2012 and March 2015. All were part of a research (including neuroimaging) protocol, aiming at the understanding of Vim RS radiobiology. All had given informed written consent. The study 
was approved by local ethics committee (in compliance with the national legislation and the Declaration of Helsinki).

\section{Tremor definition and inclusion criteria}

Essential tremor has been defined according to Elble et al.[11]. Parkinson's disease cases were excluded, as well as those patients with previous stroke or epilepsy etc, susceptible to induce structural changes. The indication for Vim RS rather than DBS were medical comorbidities drug-resistance, advanced age or patient's refusal.

\section{Gamma Knife thalamtomy}

All Vim RS procedures were all performed by same neurosurgeon (JR). After application of the Leksell ${ }^{\circledR}$ coordinate G Frame (Elekta AB, Stockholm, Sweden), under local anesthesia[30], all underwent both stereotactic CT and MRI. Indirect targeting was performed in all cases using Guiot's diagram, placed $2.5 \mathrm{~mm}$ above the AC-PC line, $11 \mathrm{~mm}$ lateral to the wall of the third ventricle, and at a mean of $7.3 \mathrm{~mm}$ in front of the PC (3.9-9.9; SD: 1.33). A unique 4-mm isocenter was used, and a maximal prescription dose of $130 \mathrm{~Gy}$.

\section{Clinical evaluation and outcome measures after Vim radiosurgery}

The same neurologist (TW) performed all clinical assessment, at baseline and 1 year after Vim RS. Tremor score on the treated hand (TSTH) was assessed according to the FahnTolosa-Marin Tremor Rating Scale [28]. It has been previously advocated that medication diminishes TSTH by approximately $50 \%[19]$ in around $50 \%$ of cases. In this sense, to be at least as effective as medication, clinical responders $(\mathrm{R})$ were considered those with at least $50 \%$ amelioration of TSTH 1 year after Vim RS, to take into account the delayed clinical effect[30]. At the time of the study, no patient was under medication.

\section{MRI acquisition and evaluation after GKT}

All imaging was obtained on a 3-T magnetic resonance imaging (MRI) scanner, SIEMENS Skyra TrioTim Scanner (Munich, Germany), with 32 channels receive array head coil. The acquisition protocol included for all cases a high-resolution, contrast-enhanced, T1weighted $(\mathrm{T} 1 \mathrm{w}), \mathrm{TR} / \mathrm{TE}=2300 / 2.03$, inversion time $900 \mathrm{~ms}$, isotropic voxel of $1 \mathrm{~mm}^{3}, 192$ slices. Images artefacted by movement were excluded. 
Statistical Parametric Mapping (SPM12) (Department of Neurology, London), performed with MATLAB 2014a, 2014 (MathWorks, Boston, MA, USA) was utilized for preprocessing and analyzing of the MRI data.

The anatomical MR images were normalized to the Montreal Neurological Institute (MNI) atlas, with voxel-size of $2 \times 2 \times 2 \mathrm{~mm}$. The normalization procedure allows the warped images to fit into a standard template brain. Furthermore, this establishes a voxel-to-voxel correspondence, between brains of different individuals. It will enable to drive group statistics and to report the results in a standard coordinate system (e.g. MNI, which is based upon data from many individuals and is fully $3 \mathrm{D}$, with data at every voxel).

They were further segmented in GM, white matter and cerebro-spinal fluid. Furthermore, they were spatially smoothed using a 8-mm full-width-at-half-maximum (FWHM) Gaussian Kernel in SPM12, to blur individual variations in gyral anatomy and to increase the signal-to-noise ratio. The smoothing procedure enables the subsequent voxel-byvoxel analysis comparable to a region of interest approach, as the voxel will contain the average concentration of gray matter from around the voxel (where the voxel is characterized by the form of the smoothing kernel). The former is so often referred as to GMD. In a structural MRI of the brain, the intensity of each volumetric pixel, or voxel, relates to the density of the gray matter in that region. The VBM technique is so comparing brain structure on a voxel-by-voxel basis. After the classical preprocessing steps, the intensity values of the voxels are compared, to identify localized differences in GMD.

The between groups SPM (T) GM maps were obtained using an analysis of variance (ANOVA) full factorial model, at a height threshold (voxel-level significance) of $\mathrm{p}<0.05$, corrected for multiple comparisons for the cluster (FWE, family-wise error method). Secondarily, if no change was initially found using FWE correction, with a less constraint voxel-threshold of $\mathrm{p}<0.005$, only corrected for the cluster volume to avoid type II errors as recommended [15]. Age, gender and the type of tremor were used as nuisance variables. Was evaluated the interaction between clinical response and time, mainly between $\mathrm{R}$ versus nonresponders (NR) patients, with the different time frame (baseline versus 1 year after Vim RS), so as to identify the eventual structural changes in GMD. The primary aim was to find differences between groups (R versus NR) in interaction with the time point (before and after Vim RS).

The SPM analysis was done by medical staff (CT, EG) not involved in the indication for treatment, Vim RS procedure or follow-up evaluation. Gray matter density was further extracted from statistically significant regions. For correlation between GMD and TSTH 
improvement, STATA version 11 (STATA Corp LLC, College Stations, TX, USA) was used, and $p$ values evaluated with Spearman correlation coefficient.

\section{RESULTS}

Thirty-one (81.6\%) patients were R and 7 (18.4\%) NR. Overall improvement in TSTH was $62.1 \%$ (mean $62.6 \%$, standard deviation 32.3 ; range $0-100 \%$ ) and in $\mathrm{R}$ subgroup was $75.2 \%$. No difference in clinical characteristics was found between R and NR (age, gender, tremor severity before Vim RS; $p>0.05$ ). No side effect was found 1 year after Vim RS.

\section{Changes in GMD between $\mathrm{R}$ and NR, during time (group factor $X$ time, figure 1)}

Changes in GMD between R and NR with time showed left temporal pole (Brodmann area, BA 38), and a larger cluster [ $\mathrm{kc}=135$ (left occipital) $>\mathrm{kc}=124$ (left temporal), indicating the number of voxels in each cluster] within the left occipital area. The former included beside the left occipital lobe (BA 19), the V4, V5, and parahippocampal place area (PPA). Figure 1 (1.1 and 1.2,) displays the boxplots with the overall value and the median for baseline and 1 year, for R and NR, by the individual cluster.

Interestingly, GMD was lower at baseline in the NR group for both regions and at 1 year had a median value similar to the R groups (which presented a slight decrease in GMD).

The table at the bottom of the figure shows the respective $p$ values at the cluster and peak level (for a detailed explanation please see the figure legend).

Furthermore, higher GMD decrease between 1 year and baseline correlated with better TSTH improvement (Spearmann= 0.01 for clusters 1 and 2, respectively; figure 1, 1.3 and 1.4). For cluster 1 (left temporal pole), higher baseline GMD predicted better improvement $($ Spearmann $=0.004$; figure 1, 1.5).

The GMD increase at 1 year after Vim RS was fairly similar for both clusters in NR, while it tended to zero in $\mathrm{R}$ (figure 1, 1.6).

\section{DISCUSSION}

To the best of our knowledge, we performed the first VBM analysis after Vim RS for drug-resistant ET. We report changes in GMD between R and NR with time within left temporal pole (BA 38) and a larger cluster including left occipital lobe (BA 19, V4, V5, and PPA), as statistically significant. Grey matter density was lower at baseline in the NR group for both regions and at 1 year had a median value similar to $\mathrm{R}$ groups (which presented a slight decrease in GMD). Higher GMD decrease between 1 year and baseline correlated with 
better TSTH improvement, for both anatomical regions. For left temporal pole, higher baseline GMD predicted better TSTH improvement.

VBM methodology has been previously used in ET (at 1.5 and $3 \mathrm{~T}$ ), with studies reporting inconsistent results[2, 4, 7-9, 16, 23], while showing no change, atrophy or volume increase in different regions. In the present study, it was beyond our purpose to analyze a healthy control group, as we were interested in how brain structurally adapts to Vim RS, in relationship to clinical response.

The involvement of left temporal pole and occipital BA 19 is a very interesting and novel finding, suggesting a distant effect of Vim RS. Left temporal pole is mainly involved in complex functions, including visual («what and where» visual pathways distinction), emotional (visual processing of emotional images, emotional attachment), processing phonological properties of written words, sign language or complex auditory processing[20]. The visual cluster actually extends beyond the BA 19, and includes parts of V4 and V5 and the PPA (posterior subdivision). Visual area V4 is part of extrastriate visual cortex[25] and is tuned for orientation, spatial frequency and color, but also long-term plasticity, stimulus salience, and is gated by signals coming from frontal-eye-fields (FEF)[31]. The former connections would be of special interest in patients with ET: in a crowded visual scene, all factors that could help distinguishing the target include color, shape and eye movements.

Vim RS is classically performed with the aim of inducing a small area of necrosis after several months, up to one year after the procedure[24]. By the expected radiobiological effect, it mimics a histologically destructive one, compared to that produced by thermocoagulation[26]. However, Ohye[22] was the first to advocate that necrotic lesion solely was not sufficient to produce tremor alleviation. Moreover, he advocated that the magnitude of the lesion induced by Vim RS was too small to account for the benefic clinical effect. The proposed «cocade theory», advocates for 4 distinct zones that might appear after Vim RS, including necrotic, subnecrotic, neuromodulatory and no effect[24].

The advantages of the VBM analysis are related to an unbiased and objective procedure, fully automated, not based on regions of interest and which is more exploratory. Furthermore, it depicts differences and/or changes (in our study) at a global and local scale. The limitations of our study are mainly related to those of the VBM method (preprocessing steps, statistical challenges etc), but also to the studied population of patients, the former including the limited sample size or the absence of a blinded-neurological assessment.

With regards to the main reasons for incomplete tremor alleviation, our experience and/or our hypothesis are : the size of the lesion (small size classically associated with more 
failures[29]), an eventual different phenotype of ET[18] (more resistant to Vim RS?), eventual adaptive changes (including structural, as depicted by VBM?), which makes the "reset" of the «tremor network» more difficult, and eventually the targeting, although we used a uniform and well-established methodology.

The role of visual association areas is reported for the first time as statistically significant and thus relevant in tremor improvement after Vim RS. How the "tremor network" (cerebello-thalamo-cortical pacemaker) modulates these structural changes in remote areas remains unknown. The anatomical connections between the primary motor cortex and visual areas might be of relevance, as previously advocated[13], for sensory guidance of movements.

\section{Conclusion}

Responders present changes in areas involved in motion, mainly locomotor monitoring towards the local and distant environment, suggesting the requirement to recruit in the targeting specific visuomotor networks.

Funding: This work has been supported by the Timone University Hospital, the Swiss National Science Foundation SNSF-205321-157040, the Centre d'Imagerie BioMédicale (CIBM) of the University of Lausanne (UNIL), the University of Geneva (UniGe), the Centre Hospitalier Universitaire Vaudois (CHUV) and the Leenaards and Jeantet Foundations. This work has been carried out thanks to the support of the A*MIDEX project ( $n^{\circ}$ ANR-11-IDEX0001-02) funded by the «Investissements d'Avenir » French Government program, managed by the French National Research Agency (ANR).

Conflict of Interest: All authors certify that they have no affiliations, with or involvement in any organization or entity with any financial interest (such as honoraria; educational grants; participation in speakers' bureaus; membership, employment, consultancies, stock ownership, or other equity interest; and expert testimony or patent-licensing arrangements), or nonfinancial interest (such as personal or professional relationships, affiliations, knowledge or beliefs) in the subject matter or materials discussed in this manuscript.

Ethical approval: The study was approved by local ethics committee of Timone University Hospital, Marseille, France. 


\section{References:}

1. Ashburner J, Friston KJ (2000) Voxel-based morphometry--the methods. NeuroImage 11:805-821

2. Bagepally BS, Bhatt MD, Chandran V, Saini J, Bharath RD, Vasudev MK, Prasad C, Yadav R, Pal PK (2012) Decrease in cerebral and cerebellar gray matter in essential tremor: a voxel-based morphometric analysis under 3T MRI. Journal of neuroimaging : official journal of the American Society of Neuroimaging 22:275-278

3. Benabid AL, Pollak P, Gao D, Hoffmann D, Limousin P, Gay E, Payen I, Benazzouz A (1996) Chronic electrical stimulation of the ventralis intermedius nucleus of the thalamus as a treatment of movement disorders. Journal of neurosurgery 84:203-214

4. Benito-Leon J, Alvarez-Linera J, Hernandez-Tamames JA, Alonso-Navarro H, Jimenez-Jimenez FJ, Louis ED (2009) Brain structural changes in essential tremor: voxelbased morphometry at 3-Tesla. Journal of the neurological sciences 287:138-142

5. Benito-Leon J, Louis ED (2006) Essential tremor: emerging views of a common disorder. Nature clinical practice. Neurology 2:666-678; quiz 662p following 691

6. Benito-Leon J, Louis ED (2007) Clinical update: diagnosis and treatment of essential tremor. Lancet 369:1152-1154

7. Buijink AW, Broersma M, van der Stouwe AM, Sharifi S, Tijssen MA, Speelman JD, Maurits NM, van Rootselaar AF (2016) Cerebellar Atrophy in Cortical Myoclonic Tremor and Not in Hereditary Essential Tremor-a Voxel-Based Morphometry Study. Cerebellum 15:696-704

8. Cerasa A, Messina D, Nicoletti G, Novellino F, Lanza P, Condino F, Arabia G, Salsone M, Quattrone A (2009) Cerebellar atrophy in essential tremor using an automated segmentation method. AJNR. American journal of neuroradiology 30:12401243

9. Daniels C, Peller M, Wolff S, Alfke K, Witt K, Gaser C, Jansen O, Siebner HR, Deuschl G (2006) Voxel-based morphometry shows no decreases in cerebellar gray matter volume in essential tremor. Neurology 67:1452-1456

10. Daniels JK, Gaebler M, Lamke JP, Walter H (2015) Grey matter alterations in patients with depersonalization disorder: a voxel-based morphometry study. Journal of psychiatry \& neuroscience : JPN 40:19-27

11. Elble RJ (2000) Diagnostic criteria for essential tremor and differential diagnosis. Neurology 54:S2-6

12. Elias WJ, Huss D, Voss T, Loomba J, Khaled M, Zadicario E, Frysinger RC, Sperling SA, Wylie S, Monteith SJ, Druzgal J, Shah BB, Harrison M, Wintermark M (2013) A pilot study of focused ultrasound thalamotomy for essential tremor. The New England journal of medicine 369:640-648

13. Glickstein M (2000) How are visual areas of the brain connected to motor areas for the sensory guidance of movement? Trends in neurosciences 23:613-617

14. Kondziolka D, Ong JG, Lee JY, Moore RY, Flickinger JC, Lunsford LD (2008) Gamma Knife thalamotomy for essential tremor. Journal of neurosurgery 108:111-117

15. Lieberman MD, Cunningham WA (2009) Type I and Type II error concerns in fMRI research: re-balancing the scale. Social cognitive and affective neuroscience 4:423428

16. Lin CH, Chen CM, Lu MK, Tsai CH, Chiou JC, Liao JR, Duann JR (2013) VBM Reveals Brain Volume Differences between Parkinson's Disease and Essential Tremor Patients. Frontiers in human neuroscience 7:247

17. Louis ED (2005) Essential tremor. The Lancet. Neurology 4:100-110 
18. Louis ED (2009) Essential tremors: a family of neurodegenerative disorders? Archives of neurology 66:1202-1208

19. Lyons KE, Pahwa R (2008) Pharmacotherapy of essential tremor : an overview of existing and upcoming agents. CNS drugs 22:1037-1045

20. Nakamura K, Kawashima R, Sugiura M, Kato T, Nakamura A, Hatano K, Nagumo S, Kubota K, Fukuda H, Ito K, Kojima S (2001) Neural substrates for recognition of familiar voices: a PET study. Neuropsychologia 39:1047-1054

21. Nicoletti V, Cecchi P, Frosini D, Pesaresi I, Fabbri S, Diciotti S, Bonuccelli U, Cosottini M, Ceravolo R (2015) Morphometric and functional MRI changes in essential tremor with and without resting tremor. Journal of neurology 262:719-728

22. Ohye C, Shibazaki T, Ishihara J, Zhang J (2000) Evaluation of gamma thalamotomy for parkinsonian and other tremors: survival of neurons adjacent to the thalamic lesion after gamma thalamotomy. Journal of neurosurgery 93 Suppl 3:120-127

23. Quattrone A, Cerasa A, Messina D, Nicoletti G, Hagberg GE, Lemieux L, Novellino F, Lanza P, Arabia G, Salsone M (2008) Essential head tremor is associated with cerebellar vermis atrophy: a volumetric and voxel-based morphometry MR imaging study. AJNR. American journal of neuroradiology 29:1692-1697

24. Regis J, Carron R, Park M (2010) Is radiosurgery a neuromodulation therapy? : A 2009 Fabrikant award lecture. Journal of neuro-oncology 98:155-162

25. Roe AW, Chelazzi L, Connor CE, Conway BR, Fujita I, Gallant JL, Lu H, Vanduffel W (2012) Toward a unified theory of visual area V4. Neuron 74:12-29

26. Schuurman PR, Bosch DA, Bossuyt PM, Bonsel GJ, van Someren EJ, de Bie RM, Merkus MP, Speelman JD (2000) A comparison of continuous thalamic stimulation and thalamotomy for suppression of severe tremor. The New England journal of medicine 342:461-468

27. Sharifi S, Nederveen AJ, Booij J, van Rootselaar AF (2014) Neuroimaging essentials in essential tremor: a systematic review. NeuroImage. Clinical 5:217-231

28. Tolosa E, Pena J (1988) Involuntary vocalizations in movement disorders. Advances in neurology 49:343-363

29. Witjas T, Carron R, Azulay JP, Regis J (2013) Gammaknife Thamamotomy For Intractable Tremors: Clinical Outcome And Correlations With Neuroimaging Features. MDS 17th International Congress of Parkinson's Disease and Movement Disorders, vol Volume 28, p 1247

30. Witjas T, Carron R, Krack P, Eusebio A, Vaugoyeau M, Hariz M, Azulay JP, Regis J (2015) A prospective single-blind study of Gamma Knife thalamotomy for tremor. Neurology 85:1562-1568

31. Zhou H, Desimone R (2011) Feature-based attention in the frontal eye field and area V4 during visual search. Neuron 70:1205-1217

Figure 1: The main effect of time point, interacting with clinical response;

Upper part: 1.1 and 1.2, global overview of GMD at baseline and 1 year for left temporal pole and visual association area, depending on clinical response $(<=50 \% \mathrm{TSTH}$ improvement, versus $>50 \%$ TSTH improvement); the boxplots reveal the range of values for GMD, as well as the median for each one of them; it is of interest that for both clusters (temporal and occipital), for a TSTH improvement $<=50 \%$, the baseline values are lower as compared with 
the ones for TSTH improvement $>50 \%$; at 1 year, the NR patients achieve a median value comparable to that of $\mathrm{R}$ at baseline, while the former remain stable; 1.3 (for the left temporal cluster) and 1.4 (for the left occipital cluster), correlations between TSTH improvement and differences in GMD (Spearmann= 0.01 for both clusters); 1.5 , correlation between baseline GMD within the left temporal cluster and TSTH improvement (Spearmann=0.004); 1.6, illustration of GMD increase (for the NR) and respectively no change (for R), as structural differences between R and NR during time, before and after Vim RS, presented by clusters (1=left temporal and 2=left occipital, with a sagittal illustration for each one of them);

Lower part, table with the main SPM results: it corresponds to the statistically significant results at cluster-level and peak-level, respectively; the overall $\mathrm{p}$ value is 0.049 ; for cluster and peak level, are reported $\mathrm{p}$ family-wise error correction (FEW), false-discovery rate (FDR), $\mathrm{Kc}=$ size of the cluster, meaning the number of the included voxels in each one fo them, and $\mathrm{p}$ unc $=$ uncorrected $\mathrm{p}$ value, as opposed to the previous (FEW and FDR); the uncorrected $\mathrm{p}$ values show statistical significance $(<0.05)$; for the peak-level, the MNI signifies Montreal Neurological Institute, which is the common space where the brains have been analyzed, so as to be able to compare them;

Lower part: and an artistic draw, illustrating the main functions for these statistically significant clusters. 